\title{
Guest Editors' Preface: The International Conference on Ultrashort High-Energy Radiation and Matter
}

The International Conference on "Ultrashort High-Energy Radiation and Matter" has been held in Villa Monastero, Varenna, Italy, during October 7-10, 2003. The conference has been jointly organized by the "Piero Caldirola" International Centre for the Promotion of Science and International School of Plasma Physics, Milan, Italy, and by the FEMTO Programme of the European Science Foundation (chaired by Prof. Charles Joachain of the University of Bruxelles).

The purpose of the conference was to be a forum to discuss the latest experimental, theoretical and computational results related to the interaction of high energy radiation with matter. High energy is intended to mean soft x-ray and beyond, but important new results from Visible systems have been incorporated.

The conference was triggered on one side by the fact that soon new bright X-ray sources will be available (X-ray Free Electron Lasers, Thomson sources), on the other by the dramatically new experiments which are already being performed using short pulse X-rays from laser sources (high harmonics, plasmas). These experiments already show the new and important possibilities offered by time resolution and high brightness.

Even if most of the papers presented in this special issue of LASER AND PARTICLE BEAMS are really related to the plasma and laser communities, in reality the conference has been interdisciplinary amongst scientists from many fields, including:

- $\mathrm{x}$-ray physics and optics

- solid state physics and material science

- biology

- quantum optics

- plasma physics

- laser physics
Topics have included, among other subjects:

1. Different X-ray sources available today: how they work and what experiments can be done:

$1.1 \mathrm{X}$-ray source development,

1.2 incoherent X-ray sources from laser-plasmas

1.3 high harmonics generation

1.4 synchrotrons

1.5 FELs

1.6 capillary X-ray sources

1.7 X-ray lasers from laser-plasmas

2. X-ray Optics Diagnostics and X-ray diagnostics

3. Photon Material Interactions

4. Plasma Physics

5. Bio Medical applications

6. Material applications

We think that one of the main success of the conference has been to put in contact three different worlds which do not often speak to each other: (i) laser and plasma physicists who have already built short-pulse high-brightness X-ray sources and realized some preliminary applications; (ii) scientists who are building the next generation X-ray FELs; and (iii) the world of possible users (biophysicists and biologists, material scientists, etc.).

Finally we would like to thank the conference secretary, Ms. Donatella Pifferetti, for her help which has been essential in order to assure the success of the conference.

Dimitri Batani University of Milano Bicocca Milano, Italy and

Alan J. Wootton Lawrence Livermore National Laboratory Livermore, California 